University of Nebraska - Lincoln

DigitalCommons@University of Nebraska - Lincoln

Insomnia characteristics and clinical correlates in Operation Enduring Freedom/Operation Iraqi Freedom veterans with posttraumatic stress disorder and mild traumatic brain injury: An exploratory study

D. M. Wallace

University of Miami Miller School of Medicine

S. Shafazand

Department of Medicine, Division of Pulmonary, Critical Care, and Sleep Medicine

\author{
A.R. Ramos \\ Bruce W. Carter Department of Veterans Affairs Medical Center \\ D.Z. Carvalho \\ Universidade Federal do Rio Grande do Sul School of Medicine \\ H. Gardener \\ University of Miami Miller School of Medicine \\ See next page for additional authors \\ Follow this and additional works at: https://digitalcommons.unl.edu/publichealthresources \\ Part of the Public Health Commons
}

Wallace, D. M.; Shafazand, S.; Ramos, A.R.; Carvalho, D.Z.; Gardener, H.; Lorenzo, D.; and Wohlgemuth, W.K., "Insomnia characteristics and clinical correlates in Operation Enduring Freedom/Operation Iraqi Freedom veterans with post-traumatic stress disorder and mild traumatic brain injury: An exploratory study" (2011). Public Health Resources. 200.

https://digitalcommons.unl.edu/publichealthresources/200

This Article is brought to you for free and open access by the Public Health Resources at DigitalCommons@University of Nebraska - Lincoln. It has been accepted for inclusion in Public Health Resources by an authorized administrator of DigitalCommons@University of Nebraska - Lincoln. 


\section{Authors}

D. M. Wallace, S. Shafazand, A.R. Ramos, D.Z. Carvalho, H. Gardener, D. Lorenzo, and W.K. Wohlgemuth 
Original Article

\title{
Insomnia characteristics and clinical correlates in Operation Enduring Freedom/Operation Iraqi Freedom veterans with post-traumatic stress disorder and mild traumatic brain injury: An exploratory study
}

\author{
D.M. Wallace ${ }^{\mathrm{a}, \mathrm{b}, *}$, S. Shafazand ${ }^{\mathrm{c}}$, A.R. Ramos ${ }^{\mathrm{b}}$, D.Z. Carvalho ${ }^{\mathrm{d}}$, H. Gardener $^{\mathrm{a}}$, D. Lorenzo ${ }^{\mathrm{a}, \mathrm{b}}$, \\ W.K. Wohlgemuth ${ }^{\mathrm{a}, \mathrm{b}}$ \\ ${ }^{a}$ Department of Neurology, University of Miami Miller School of Medicine, Miami, FL, USA \\ ${ }^{\mathrm{b}}$ Department of Neurology, Bruce W. Carter Department of Veterans Affairs Medical Center, Miami, FL, USA \\ ${ }^{\mathrm{c}}$ Department of Medicine, Division of Pulmonary, Critical Care, and Sleep Medicine, Miami, FL, USA \\ ${ }^{\mathrm{d}}$ Neurology Service, Hospital de Clínicas de Porto Alegre, Universidade Federal do Rio Grande do Sul School of Medicine, Porto Alegre, RS, Brazil
}

\section{A R T I C L E I N F O}

\section{Article history:}

Received 6 April 2011

Received in revised form 20 May 2011

Accepted 1 June 2011

Available online 16 September 2011

\section{Keywords:}

Veterans

Traumatic brain injury

Insomnia

Post-traumatic stress disorder

Actigraphy

Sleep disturbances

\begin{abstract}
A B S T R A C T
Background: There is limited data on chronic insomnia in Operation Enduring Freedom/Operation Iraqi Freedom (OEF/OIF) veterans, in whom post-traumatic stress disorder (PTSD) and mild traumatic brain injury (mTBI) often co-exist. Our aim was to compare sleep characteristics of three groups of OEF/OIF veterans: (1) healthy sleepers (HS), (2) those with insomnia associated with PTSD and mTBI (PTSD-mTBI), and (3) those with insomnia associated with PTSD alone.

Methods: Consecutive veterans with insomnia complaints (>6 months) were recruited over 6 months from the Miami VA Post Deployment clinic. Participants completed a sleep disorders clinical interview, medical history, and questionnaires about insomnia, sleepiness, pain, fatigue, depression, PTSD, and health-related quality of life. They underwent polysomnography (PSG) with 2 weeks of actigraphy (ACT) and sleep diaries.

Results: There were no differences in demographics or most questionnaire responses between PTSD and PTSD-mTBI groups. Subjective daytime sleepiness was significantly greater in PTSD-mTBI subjects compared with HS and PTSD participants. Significant co-morbid sleep disorders were noted in insomnia patients. PSG and ACT wake after sleep onset was significantly shorter in PTSD-mTBI subjects as compared with PTSD participants.

Conclusion: Insomnia patients with PTSD-mTBI were subjectively sleepier despite spending less time awake during the night than PTSD subjects, possibly as a consequence of head trauma.
\end{abstract}

Published by Elsevier B.V.

\section{Introduction}

Insomnia, frequent difficulty with falling or staying sleep or early morning awakenings resulting in daytime impairments, is one of the most common complaints reported by Operation Enduring Freedom/Operation Iraqi Freedom (OEF/OIF) veterans at postdeployment clinics [1-4]. Most patients recover fully within 36 months after mild traumatic brain injury (mTBI); however, 10$15 \%$ of patients have persistent post-concussive symptoms (headaches, anxiety, depression, disequilibrium, cognitive complaints, and insomnia) [5]. Acutely, sleep disruption from insomnia or other sleep disorders may mediate entrenchment of post-trau- matic stress disorder (PTSD) and post-concussive symptoms by impeding restorative processes occurring during undisturbed sleep [6,7]. Furthermore, insomnia after mTBI or PTSD may initiate or exacerbate psychiatric problems, behaviour, memory and social functioning, and negatively impact rehabilitative treatments. Insomnia with short sleep duration has also been associated with worse quality of life, increased health-care costs, increased risk for hypertension, diabetes and all-cause mortality [8-11]. Although PTSD and mTBI often co-exist in this veteran population [3], there is limited information about clinical correlates and insomnia characteristics in OEF/OIF veterans with PTSD and mTBI $[1,2,4,12,13]$.

\subsection{Insomnia and $m T B I$}

* Corresponding author at: Bruce W. Carter Department of Veterans Affairs Medical Center, 1201 NW 16th Street, Miami, FL 33125, USA. Tel.: +1 3055753192; fax: +1 3055753210 .

E-mail address: dwallace@med.miami.edu (D.M. Wallace).
Chronic insomnia, insomnia lasting for more than 1 month, can be the heralding symptom of PTSD and may develop after mTBI 
$[2,7]$. The prevalence of insomnia complaints after mTBI ranges widely from $21 \%$ to $93 \%$ [3-5,14,15]. Insomnia after mTBI is complicated and multifactorial, possibly resulting from injury to cerebral structures responsible for regulating sleep, co-morbid affective and anxiety disorders, post-traumatic pain, and the side effects of multiple medications used to treat some of these co-morbid conditions [7]. Polysomnography (PSG) studies in insomnia associated with mTBI have shown variable abnormalities including sleep macrostructure disturbances correlating with subjective complaints. These include a greater proportion of 'light sleep', higher sleep fragmentation with greater wake after sleep onset (WASO) and increased number of awakenings as compared with healthy sleepers (HSs) [16-18]. Actigraphic studies in mTBI are limited but have corroborated these PSG findings and demonstrated greater time spent in bed and increased total sleep time (TST) as compared with controls [18]. While comparisons between these studies are difficult due to methodological differences, all of these findings suggest sleep instability after mTBI.

\subsection{Insomnia and PTSD}

PTSD is characterised by three symptom clusters (re-experiencing, avoidance, and hyperarousal) that can all contribute to insomnia. Recurring military-related nightmares, avoiding sleep because of these, and nocturnal checking 'safety perimeter' behaviours (frequent checking of windows and doors) in the home environment are all incongruent with sleep initiation. PSG studies demonstrating objective sleep abnormalities in PTSD have yielded inconsistent results. A meta-analysis of 20 PSG-based studies in patients with PTSD revealed that increased rapid eye movement (REM) density, greater N1 sleep and decreased slow-wave sleep were seen in PTSD patients compared with normals [19]. Other consistent findings have involved dysfunction of REM sleep mechanisms including increased phasic events (motor events) and REM sleep fragmentation [20-23]. These abnormalities support findings of centrally measured hyperarousal during sleep in PTSD [24]. Some investigators have found co-morbid mild sleep-disordered breathing (SDB) to be common in patients with PTSD while others have not $[23,25]$. Actigraphic data in PTSD is limited but a few studies have used it to estimate TST and awakenings in veterans with PTSD [26].

Although young veterans may suffer from a wide variety of sleep complaints including insomnia, snoring and nocturnal behaviours, the necessity for a comprehensive sleep evaluation is not well established. Among these sleep disorders, insomnia is the most prevalent [2]. Improved understanding of the nature of insomnia and its clinical correlates in OEF/OIF veterans may help identify potential interventional targets (behavioural and pharmacological) and determine need for sleep testing. Most studies of returning OEF/OIF veterans' sleep quality have relied on responses to a single-item regarding sleep embedded within an instrument measuring other outcomes (depression and PTSD) and have not employed validated sleep questionnaires, laboratory PSG or repeated objective measures in home environments [2-5,13]. Selfreported measures are problematic as lone sleepers may lack awareness of occult sleep disturbances. Repeated home assessments may be useful as persons with insomnia may become conditioned to their bedroom environment and have high night-to-night variability [27]. A single night of laboratory PSGs may fail to reveal these abnormalities. In this study, we measure the sleep of combat veterans subjectively reporting chronic insomnia symptoms (i.e., difficulty falling or staying asleep or early morning awakenings for at least 6 months with daytime repercussions) not attributable to another primary sleep disorder (i.e., significant sleep apnoea or restless legs syndrome) using comprehensive sleep evaluations. The primary aim of this prospective study is to assess sleep parameters using validated questionnaires and structured clinical inter- views for sleep disorders, PSG, actigraphy (ACT), and sleep diaries (SDs). These parameters were compared amongst veterans with PTSD, veterans with PTSD-mTBI, and HSs. We hypothesised that veterans with PTSD-mTBI would have more severe insomnia complaints, show greater PSG and ACT sleep fragmentation and report worse quality of life compared with veterans with PTSD alone and HSs.

\section{Methods}

\subsection{PTSD and PTSD-mTBI Subjects}

Subjects with PTSD or PTSD-mTBI were consecutive OEF/OIF veterans with insomnia complaints ( $\geqslant 6$ months) recruited from the Miami VA HealthCare System Post Deployment clinic during routine clinical visits between 1st June and 31st December 2009 (control subjects are described in next section). In this study, insomnia was defined as difficulty initiating or maintaining sleep or with early morning awakenings on more than three nights of the week for at least 6 months despite adequate opportunity to sleep. Subjects also had to endorse at least one accompanying daytime symptom (fatigue, sleepiness, mood disturbance, poor motivation, concentration difficulties, or worries about sleep) [28]. Sleep pattern was documented during 2 weeks of SDs. Inclusion criteria were (1) adults aged 18-50 years, (2) OEF/OIF veterans with combat exposure with stable physical and mental health, and (3) English literacy at grade 5 or higher. Subjects were excluded if (1) prior to deployment, they had history of sleep disorder, psychiatric disorder or a TBI, (2) suffered penetrating, moderate or severe TBI, (3) had active substance abuse, (4) had insomnia secondary to pain or another primary sleep disorder (moderate to severe SDB, periodic limb movement disorder or moderate to severe restless legs syndrome), (5) were using medication causing insomnia (i.e., stimulant medications), or (6) failed to complete all study assessments. Subjects using stable doses (unchanged dosage for 3 months preceding enrollment) of hypnotic medications were not excluded from the study if their insomnia complaints persisted despite medication use.

\subsection{Inclusion criteria for the control group}

Consecutive OEF/OIF veterans were screened for sleep disorders and typical sleep duration during their routine well visits to the Miami VA Health Care System Post Deployment primary care provider. They were required to be in good physical and mental health, with no sleep complaints, free of medications influencing sleep and having sufficient, regular sleeping hours. Thirty-six veterans were screened with a brief structured clinical interview for sleep disorders and review of their medical record for inclusion and exclusion criteria. Six HSs were identified.

The study was approved by the Bruce W. Carter Department of Veterans Affair Medical Center institutional review board and written patient consent was obtained from all study participants.

\subsection{Procedure}

All subjects were scheduled for a 4-h baseline study visit, where they completed a structured clinical interview for sleep disorders, a full neurological examination, comprehensive lifetime concussion history and standardised instruments for assessment of mental health, sleep disorders, and health-related quality of life (HRQOL), as described below. Demographic characteristics, medication use (including those prescribed for insomnia) and military service details (including exposure to traumatic events such as explosions, history of head trauma or other injuries occurring 
during deployment) were obtained through questionnaires and review of medical records. Information was corroborated by DD214s forms, which provide information about deployment length, military occupation, and discharge.

\subsection{Clinical assessment of insomnia and other sleep disorders}

Subjects completed the Duke Structured Interview for Sleep Disorders (DSISD), which is derived from the International Classification of Sleep Disorders (ICSD-2) [28]. Using this instrument allowed the identification of insomnia subtype and other comorbid sleep disorders. When available, bed partners helped corroborate reports.

\subsection{Self-report measures}

\subsubsection{Post-traumatic stress checklist-military version (PCL-M)}

The post-traumatic stress checklist-military version (PCL-M) is a 17-item National Center for PTSD Checklist used to assess the severity of avoidance, hyperarousal, and intrusion symptoms [29]. The PTSD checklist is based on well-validated case definitions used in veteran and military populations [1]. The PCL-M scores range from 0 to 85, with higher scores indicative of more severe symptoms. Participants were defined as having PTSD symptoms if they endorsed at least three avoidance symptoms, two hyperarousal symptoms and one intrusion symptom at 'moderate' or higher levels and scored at least 50 on the PTSD checklist, indicative of significant distress.

\subsubsection{Insomnia severity index (ISI)}

The insomnia severity index (ISI) is a brief, seven-item instrument measuring the patient's perception of his or her insomnia. Items assess the severity of sleep onset and sleep maintenance difficulties (both nocturnal and early morning awakenings), satisfaction with current sleep pattern, interference with daily functioning, noticeability of impairment attributed to the sleep problem, and degree of distress or concern caused by the sleep problem. The total scores range from 0 to 28 , with a cut-off score of 8 suggestive of mild clinical insomnia and higher scores consistent with worse insomnia symptoms. Its internal consistency, concurrent validity, and sensitivity to clinical improvements are well established [30].

\subsubsection{Epworth sleepiness scale (ESS)}

The Epworth sleepiness scale (ESS) is a measure of subjective daytime sleepiness used for patients with SDB. Eight items are rated on a scale of $0-3$, total scores range $0-24$, with higher scores indicating a greater propensity to fall asleep in different situations. A score $\geqslant 10$ indicates excessive daytime sleepiness. In samples of SDB patients, it shows high internal consistency and correlates well with objective measures of sleep latency [31].

\subsubsection{Fatigue severity scale (FSS)}

The fatigue severity scale (FSS) is a nine-item scale originally developed to capture an individual's experience of mental or psychological fatigue and how it interferes with performing daily activities (i.e., exercise and work) over the prior week. A cut-off score of $\geqslant 36$ defines fatigue. It has been used and validated in diverse neurologic patient populations [32].

\subsubsection{Beck depression inventory-II (BDI-II)}

This measure consists of 21 items evaluating depressive symptoms experienced in the prior 2 weeks. Total scores range from 0 to 62 (0-13 no depression; 14-21 mild depression; 22-30 moderate depression, and $>30$ severe depression). It is widely used and its psychometric properties have been well documented [33].

\subsubsection{Pain assessment}

Participants were asked to rate "How much does pain interfere with your nightly ability to begin or continue sleeping?" on a $100-$ $\mathrm{mm}$ visual analogue scale. One end of the scale was labelled " "not at all" and the other end labelled "extremely".

\subsubsection{Short Form-36 (SF-36)}

The Short Form-36 (SF-36) is a 36-item quality-of-life measure that assesses eight domains: (1) physical functioning; (2) role limitation due to physical health problems (role physical); (3) bodily pain; (4) general health perceptions; (5) vitality; (6) social functioning; (7) role limitations due to emotional health problems (role emotional); and (8) mental health [34]. Physical function and role physical scales best distinguish between groups differing in severity of chronic medical conditions and have the purest interpretation with regard to physical health; mental health and role-emotional scales best distinguish between groups differing in severity of psychiatric disorders and have the purest interpretation with regard to mental health. Social function, vitality, and general health perception scales measure both physical, and mental health status [34]. All health measures were scored on scales of $0-100$, with higher scores indicating better health. The SF-36 has been carefully tested, validated, and used in patients with chronic diseases including chronic insomnia $[8,35]$.

\subsection{Clinical assessment of traumatic brain injury}

All participants underwent a full neurological exam by a boardcertified neurologist experienced with TBI populations (DMW). A lifetime concussion history was taken including history of childhood accidents, falls, motor vehicle accidents, and participation in contact sports. Participants completed questionnaires concerning head trauma occurring during deployment including (1) loss of consciousness and post-traumatic amnesia duration, (2) aetiology, (3) post-concussion symptoms, (4) date, and (5) number of events. Mild TBI diagnosis was made per American Academy of Neurology Grade III concussion guidelines, which require loss of consciousness (LOC) $\leqslant 30 \mathrm{~min}$ [36]. When available, mTBI diagnosis was corroborated with documentation from active military records. As military medical records of a TBI incident were sometimes not available, the participant's account of the event was used to satisfy criteria.

\subsection{Nocturnal PSG}

A standard montage was used, including electroencephalographic (EEG), electromyographic (EMG), and electrooculographic (EOG) monitoring for an in-lab overnight video-PSG. Measures of airflow and EMG of the legs and arms were taken to detect SDB and limb movements. Participants were asked to return to the sleep lab for a second night of evaluation within a week.

The second PSG was used to determine sleep stages, arousals and sleep-related events and were scored according to established American Academy of Sleep Medicine criteria by an experienced technician blinded to subject's group assignment [37]. Sleep onset latency (SOL) was measured as the time between lights out to the first 10 min of persistent sleep. Awakenings were counted when two consecutive epochs were scored as wake. REM latency represents the time between sleep onset and the first REM episode noted.

Upon awakening after each night of PSG, participants were asked to complete a short questionnaire approximating their time in bed (TIB), TST, SOL, and WASO and comparing their sleep quality in the sleep lab to their sleep at home.

A preliminary analysis (Wilcoxon signed-ranks test) on PSG measures (sleep efficiency (SE), SOL, and WASO) was computed 
to verify whether participants slept differently in the sleep laboratory on night 1 compared with night 2 (first-night effect). Neither insomnia group had significant differences in SOL between PSG nights, but the HS group had significantly higher SOL on night 1 . Both HS and PTSD-mTBI participants had significantly lower SE and higher WASO on night 1 , suggesting that they had a "first night effect". Hence, as is commonly done in sleep research, only night 2 was analysed further.

\subsection{ACT/home SDs}

On the second night of PSG, participants were given an actigraph (Mini Mitter-Respironics Inc., Bend, OR, USA; Actiwatch Spectrum) to wear on the non-dominant wrist. An actigraph is a wristwatch-like device that records movement through an accelerometer and has a photodiode for detecting light intensity exposure. It can be used to infer whether the subject is asleep or awake. PSG and ACT software were synchronised to correlate sleep variables measured by both assessments. Participants were asked to wear the actigraph for 14 consecutive days and nights. In addition, they were asked to maintain an SD, which they were instructed to fill out every morning upon awakening, noting times they went to bed, number and length of nocturnal awakenings, morning wake up times, quality of the previous night's sleep and any naps taken during the day. At the end of the 2-week study period, the actigraph and SD were returned to the sleep centre.

ACT data were downloaded and analysed using Actiware 5.0 software and Actiwatch Firmware version 01.01.0007 (Mini Mitter-Respironics Inc., Bend, OR, USA) by an experienced scorer (DMW). Data were averaged into 1-min epochs. The daily primary sleep periods were calculated manually in conjunction with SD data and actigraphically recorded light information. The wake threshold value (i.e., the number of activity counts used to define wake) was set to medium sensitivity of 40.0 activity counts per epoch. The sleep onset, offset, and ACT-TST were recorded and SE was calculated. ACT-SOL was defined as the start of the first 10 min of consecutive sleep after bedtime. SD and photodiode 'lights off' data were used to determine each bedtime. Similarly, sleep offset was defined as the end of the last $10 \mathrm{~min}$ of consecutive sleep prior to wake time.

Measures of sleep fragmentation on ACT were: (1) total WASO, (2) number of nocturnal awakenings lasting at least $5 \mathrm{~min}$ (NA $\geqslant 5$ min) during the primary sleep interval, and (3) the sleep fragmentation index (the ratio of the number of groups of consecutive epochs of sleep to the total number of sleep epochs such that a greater index indicates less continuous sleep).

Home SDs provided the following subjective measurements of participant's sleep: TIB (SD-TIB), TST (SD-TST: sleep duration in the last $24 \mathrm{~h}$ ), SE (SD-SE), SOL (SD-SOL), WASO (SD-WASO), number of nocturnal awakenings and sleep quality (Likert scale 1 'very poor"' to 5 'excellent').

\subsection{Data analysis}

Clinical characteristics, questionnaires, and PSG variables were compared between the PTSD, PTSD-mTBI, and HS groups. Results are presented as mean \pm standard deviation or median (interquartile range) according to their normality distribution. Groups were compared by Fisher-Freeman-Halton exact test, analysis of variance (ANOVA), and Kruskal-Wallis for categorical and continuous (parametric or non-parametric) data, respectively. If significant differences were shown, they were followed by a pair-wise Fisher's exact test with Bonferroni's correction and post hoc analyses with Tukey's test and Conover-Inman's test, respectively. Cliff's delta $(\delta)$ statistic was applied to pair-wise comparisons to estimate non-parametric effect sizes. It describes the percentage of non- overlap between two sample distributions, where the sign reflects the direction of the comparison (negative when the second comparison group has higher values than the first) [38]. Hence, a $\delta=0.75$ for a comparison A versus B means a $75 \%$ non-overlap (25\% overlap) between samples distributions where the first group A has greater values than those of group B. Therefore, $\delta$ ranges from 0 (complete overlap) to \pm 1 (no overlap) [38-40]. Cohen's interpretations of the effect size index $d$ in terms of the non-overlap between two normal distributions provide a direct bridge between $d$ and Cliff's delta $(\delta)$ so that a $d$ effect size of 0.20 (small) will have a $\delta$ of 0.147 , a $d$ effect size of 0.50 (medium) corresponds to a $\delta$ of 0.33 and a $d$ effect size of 0.80 (large) corresponds to a $\delta$ of 0.474 [38]. Cliff's delta statistics has been shown to be a robust and reliable effect size measure when parametric assumptions cannot be met [38-40].

Spearman's rank correlation was used to determine relationships between TST, SE, SOL, and WASO between PSG, SD, and ACT recordings on the second PSG night. Statistical significance was assumed for two-tailed $p$-values $<0.05$. Statistical analyses were performed with Statistical Package for the Social Sciences (SPSS) Statistics 17.0 (SPSS, Chicago, IL, USA), StatsDirect 2.7.2 (StatsDirect Ltd., UK), and ViSta 6.4 (Visual Statistics System, Chapel Hill, NC, USA) with an Effect Size Calculator plugin (ES-Calc.) [40].

\section{Results}

\subsection{Demographics and clinical characteristics}

Thirty-eight male veterans were recruited into the study. Eight were excluded (two for moderate-severe obstructive sleep apnoea, one for severe restless legs symptoms, one for paradoxical insomnia, one for history of mTBI predating deployment, and three failed to complete all procedures). Six HS, nine PTSD patients, and 15 PTSD-mTBI patients completed the study. The mean age of the 30 subjects was $31 \pm 7$ years and the mean body mass index (BMI) was $27 \pm 4 \mathrm{~kg} \mathrm{~m}^{-2}$. Subject ethnicity was predominantly Hispanic (63\%), followed by $23 \%$ Caucasian, $7 \%$ Asian, and $7 \%$ AfricanAmerican. There were no significant differences between the groups in mean age, mean education years, mean time since deployment completion, or mean length or number of deployments. Employment, marital status, sleeping arrangement, and coffee, alcohol, or tobacco consumption distribution also did not differ between the groups (Table 1).

The PTSD and PTSD-mTBI groups used a significantly higher mean number of hypnotics when compared with HS (Table 1). All insomnia patients were using at least one psychotropic medication. The PTSD group had a significantly higher use of analgesics compared with the HS group (78\% vs. $0 \%, p=0.021$, Fisher's exact test with Bonferroni's correction). There was no significant difference between PTSD and PTSD-mTBI groups in medication regimen.

The mean number of mTBIs was $2.1 \pm 1.3$ events. Eighty-two percent of $\mathrm{mTBI}$ reported LOC for less than $5 \mathrm{~min}$. Aetiologies of mTBIs were blast injury in $15 \%$ and mixed (blast followed by motor vehicle accident, shrapnel injury or fall) in $85 \%$. All PTSD-mTBI subjects had non-contrast magnetic resonance imaging (1.5 Tesla) which was unremarkable for intracranial abnormalities.

\subsection{Questionnaires}

The HS group had significantly lower median scores on questionnaires concerning insomnia symptoms, fatigue, pain, depression, and PTSD and significantly higher HRQOL scores compared with both insomnia groups (Table 2 ). Insomnia severity proportion was not significantly different in the PTSD and PTSD-mTBI groups 
Table 1

Data presented as means $\pm \mathrm{SD}^{*}$ or frequency (\%), as appropriate. Post hoc significance levels are labeled as follows.

\begin{tabular}{|c|c|c|c|}
\hline & $\mathrm{HS}(n=6)$ & PTSD $(n=9)$ & PTSD-mTBI $(n=15)$ \\
\hline \multicolumn{4}{|l|}{ Demographics } \\
\hline Age (years)* & $31 \pm 7$ & $30 \pm 7$ & $31 \pm 8$ \\
\hline Education (years) ${ }^{*}$ & $14 \pm 2$ & $14 \pm 2$ & $14 \pm 1$ \\
\hline BMI $\left(\mathrm{kg} / \mathrm{m}^{2}\right)$ & $26 \pm 1$ & $28 \pm 5$ & $28 \pm 4$ \\
\hline Return to US (months)* & $29 \pm 10$ & $35 \pm 19$ & $30 \pm 18$ \\
\hline Deployments $(n, \%)$ & $1.3 \pm 0.5$ & $1.3 \pm 0.5$ & $1.5 \pm 0.6$ \\
\hline Employed (n, \%) & $4(67)$ & $6(67)$ & $5(33)$ \\
\hline Full-time & $2(33)$ & $4(44)$ & $4(27)$ \\
\hline Shift-work $(n, \%)$ & $3(50)$ & $5(56)$ & $11(73)$ \\
\hline \multicolumn{4}{|l|}{ Marital status ( $n, \%)$} \\
\hline Single & $2(33)$ & $1(11)$ & $6(40)$ \\
\hline Married & $4(67)$ & $5(56)$ & $4(27)$ \\
\hline Divorced/separated & $0(0)$ & $3(33)$ & $5(33)$ \\
\hline \multicolumn{4}{|c|}{ Sleeping arrangement $(n, \%)$} \\
\hline Alone & $2(33)$ & $3(33)$ & $11(73)$ \\
\hline With partner & $4(67)$ & $6(67)$ & $4(27)$ \\
\hline \multicolumn{4}{|l|}{ Habits } \\
\hline Caffeine (drinks/day)* & $1.0 \pm 0.6$ & $2.2 \pm 1.8$ & $1.9 \pm 2$ \\
\hline Alcohol (drinks/week) ${ }^{*}$ & $1.7 \pm 1$ & $1.6 \pm 2.2$ & $2.3 \pm 3.3$ \\
\hline \multicolumn{4}{|l|}{ Smoking $(n, \%)$} \\
\hline Never & $4(67)$ & $8(89)$ & $10(67)$ \\
\hline Past & $1(17)$ & $0(0)$ & $1(7)$ \\
\hline Current & $1(17)$ & $1(11)$ & $4(27)$ \\
\hline \multicolumn{4}{|l|}{ Medications (n, \%) } \\
\hline Hypnotics $(n)$ & $0^{\mathrm{a}}$ & $1.3 \pm 1$ & $1.0 \pm 1$ \\
\hline Benzodiazepines & 0 & $3(33)$ & $2(13)$ \\
\hline Zolpidem & 0 & $2(22)$ & $4(27)$ \\
\hline Quetiapine & 0 & $2(22)$ & $5(33)$ \\
\hline Trazodone & 0 & $3(33)$ & $1(7)$ \\
\hline \multicolumn{4}{|l|}{ Antidepressants } \\
\hline SSRIs & 0 & $5(56)$ & $7(47)$ \\
\hline SNRIs & 0 & $1(11)$ & $1(7)$ \\
\hline TCA & 0 & $3(33)$ & $5(33)$ \\
\hline Bupropion & 0 & $3(33)$ & $1(7)$ \\
\hline Stabilizers/AEDs & 0 & $5(56)$ & $6(40)$ \\
\hline Analgesics & $0^{\mathrm{b}}$ & $7(78)$ & $6(40)$ \\
\hline
\end{tabular}

SSRI, selective serotonin reuptake inhibitor; SNRI, serotonin-norepinephrine reuptake inhibitor; TCA, tricyclic antidepressant; AED, antiepileptic drug.

a $p<0.01$ for comparison between HS and both insomnia groups.

b $p<0.05$ for comparison between HS and PTSD.

with $44 \%$ and $60 \%$ reporting moderate insomnia and 56\% and 40\% reporting severe insomnia symptoms, respectively. Seventy-eight percent of PTSD and $80 \%$ of PTSD-mTBI subjects complained of 'se- vere' or 'very severe' sleep-initiation difficulties; $89 \%$ of PTSD and $87 \%$ of PTSD-mTBI complained of 'severe' or 'very severe' sleepmaintenance difficulties; and 44\% of PTSD and 53\% of PTSD-mTBI complained of 'severe' or 'very severe' problems with early awakenings. The median ESS score of the PTSD-mTBI group (14 (interquartile range, IQR, 9)) was significantly greater than that of the PTSD (6 (IQR 7)) and HS (5 (IQR 3)) groups. The prevalence of daytime sleepiness (ESS $\geqslant 10$ ) in the HS, PTSD, and PTSD-mTBI groups was $0 \%, 33 \%$, and $60 \%$, respectively. None of the HSs reported significant daytime fatigue (FSS $\geqslant 36$ ) as compared with $89 \%$ and $73 \%$ of the PTSD and PTSD-mTBI groups, respectively. All patients with PTSD reported co-morbid depressive symptoms. Distribution of depression symptom severity between the PTSD and PTSD-mTBI groups was not significantly different with $22 \%$ and $20 \%$ reporting mild depression, $22 \%$ and $40 \%$ reporting moderate depression, and $56 \%$ and $40 \%$ reporting severe depression symptoms, respectively. There were no significant differences in any of the questionnaires' median scores between the PTSD and PTSD-mTBI groups (Table 2).

\subsection{Co-morbid sleep disorders}

A variety of REM and non-REM (NREM) parasomnia events were prevalent in the insomnia groups (Table 3 ). Nine subjects reported at least one REM or NREM parasomnia. One participant reported several incidents of zolpidem-associated parasomnia. REM sleep behaviour disorder was detected in four patients with excessive EMG tone noted during REM sleep associated with history of dream-enactment complaints and sleep-related injury. All of these patients were using selective serotonin reuptake inhibitor (SSRI) antidepressants. Weekly nightmares were significantly more common in PTSD and PTSD-mTBI groups compared with the HS group. Four PTSD-mTBI and three PTSD subjects had REM-related obstructive respiratory events (REM-AHI $\geqslant 15$ ). There were no significant differences in mild SDB, parasomnias or sleep-related movement disorders between the insomnia groups.

\subsection{Polysomnography}

Several differences were noted in sleep initiation and maintenance measures and sleep architecture between the HS, PTSD, and PTSD-mTBI groups (Table 4). The PTSD and PTSD-mTBI groups' median TST and SE were significantly lower compared with

Table 2

Data presented as medians (interquartile range). Post hoc significance levels are labeled as follows:

\begin{tabular}{|c|c|c|c|c|c|c|}
\hline & \multirow[t]{2}{*}{ HS (A) $(n=6)$} & \multirow[t]{2}{*}{ PTSD (B) $(n=9)$} & \multirow[t]{2}{*}{ PTSD-mTBI $(C)(n=15)$} & \multicolumn{3}{|c|}{ Cliff's delta $(\delta)^{\dagger}$} \\
\hline & & & & $\mathrm{AxB}$ & $\mathrm{AxC}$ & $\mathrm{BxC}$ \\
\hline ISI & $5(3)^{a}$ & $22(5)$ & $20(8)$ & -1 & -1 & -0.02 \\
\hline ESS & $5(3)^{b}$ & $6(7)$ & $14(9)^{\mathrm{c}}$ & -0.52 & -0.79 & -0.56 \\
\hline FSS & $18(7)^{a}$ & $46(20)$ & $47(22)$ & -1 & -1 & 0.63 \\
\hline BDI-II & $3(5)^{a}$ & $33(26)$ & $26(17)$ & -1 & -1 & 0.04 \\
\hline PCL-M & $7(7)^{a}$ & $62(22)$ & $56(18)$ & -1 & -1 & 0.05 \\
\hline Pain VAS & $0(1)^{a}$ & $30(32)$ & $10(20)$ & -0.61 & -0.68 & 0.24 \\
\hline SF-36 total score & $95(13)^{a}$ & $35(44)$ & $30(25)$ & 1 & 1 & 0.07 \\
\hline Physical functioning & $100(0)^{a}$ & $50(40)$ & $55(45)$ & 1 & 1 & -0.19 \\
\hline Role physical & $100(0)^{a}$ & $0(100)$ & $0(50)$ & 0.68 & 1 & 0.19 \\
\hline Bodily pain & $100(10)^{a}$ & $22(36)$ & $22(29)$ & 0.94 & 0.99 & 0.06 \\
\hline General health & $95(33)^{a}$ & $42(27)$ & $35(30)$ & 0.69 & 0.73 & 0.27 \\
\hline Vitality & $85(14)^{a}$ & $25(33)$ & $25(25)$ & 0.98 & 0.98 & 0.01 \\
\hline Social functioning & $100(0)^{a}$ & $38(56)$ & $25(38)$ & 1 & 1 & 0.22 \\
\hline Role emotional & $100(0)^{a}$ & $0(50)$ & $0(0)$ & 0.89 & 0.93 & 0.15 \\
\hline Mental health & $82(29)^{a}$ & $40(40)$ & $36(20)$ & 0.94 & 0.9 & -0.1 \\
\hline
\end{tabular}

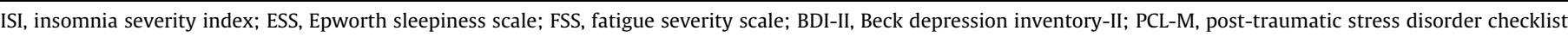
military version; VAS, visual analogue scale; SF-36, Short Form-36.

a $p<0.05$ for comparison between HS and both insomnia groups;

b $p<0.01$ for comparison between HS and PTSD-mTBI;

c $p<0.05$ for comparison between PTSD and PTSD-mTBI.

${ }^{\dagger}$ Effect size estimate: $\delta=0.147$ (small); $\delta=0.33$ (moderate); and $\delta=0.474$ (large). 
Table 3

Data represented as frequency (\%). Post hoc significance levels are labeled as follows:

\begin{tabular}{|c|c|c|c|}
\hline & $\mathrm{HS}(n=6)$ & $\operatorname{PTSD}(n=9)$ & $\begin{array}{l}\text { PTSD-mTBI } \\
(n=15)\end{array}$ \\
\hline \multicolumn{4}{|l|}{ Sleep disorders (n, \%) } \\
\hline $\operatorname{SDB}(A H I \geqslant 5)$ & 0 & $1(11)$ & $4(27)$ \\
\hline \multicolumn{4}{|l|}{ Parasomnias } \\
\hline Confusional arousals & 0 & $4(44)$ & $4(27)$ \\
\hline Sleepwalking & 0 & $2(22)$ & $3(20)$ \\
\hline SRED & 0 & $1(11)$ & $1(7)$ \\
\hline RBD & 0 & $1(11)$ & $3(20)$ \\
\hline $\begin{array}{l}\text { Sleep-related } \\
\text { hallucinations }\end{array}$ & 0 & $3(33)$ & $2(13)$ \\
\hline Nightmares & $1(17)^{a}$ & $8(89)$ & $12(80)$ \\
\hline \multicolumn{4}{|c|}{ Sleep-related movement disorders } \\
\hline Bruxism & $2(33)$ & $6(67)$ & $12(80)$ \\
\hline RLS & 0 & $2(22)$ & $2(13)$ \\
\hline PLMS & 0 & $1(11)$ & $1(7)$ \\
\hline
\end{tabular}

SDB, sleep disordered breathing; AHI, apnoea-hypopnea index; SRED, sleep-related eating disorder; RBD, REM sleep behaviour disorder; RLS, restless legs syndrome; PLMS, periodic limb movements in sleep.

${ }^{a} p<0.05$ for comparison between HS and both insomnia groups.

the HS group, whereas the PTSD and PTSD-mTBI groups' median SOL, number of awakenings, arousal index, and percentage of N1 sleep were significantly higher than those for the HS group. Percentage of N2, N3 (slow-wave sleep), and REM sleep was similar in the three groups. The PTSD group had significantly longer median WASO values as compared with the PTSD-TBI group (49 (IQR 35) vs. 15 (IQR 41) min, $p=0.04$, Conover-Inman's test). Other measures of sleep continuity (SE, arousal index and awakenings) were similar between the insomnia groups. There were no other significant differences in sleep architecture between the PTSD and PTSD-mTBI groups for other PSG variables.

Second-night PSG and ACT were significantly correlated for TST, $\mathrm{SE}$, and WASO in insomnia groups and the HS group. SOL was significantly correlated for the HS group and PTSD-mTBI but not the PTSD group.

In the post-PSG diary, five of six HS, one of nine PTSD and three of 15 PTSD-mTBI subjects reported similar in-lab sleep quality as compared to home sleep quality. Seven PTSD and nine PTSD-mTBI subjects reported improved in-lab sleep quality while one HS, one PTSD and three PTSD-mTBI subjects reported worse in-lab sleep quality as compared with home sleep quality. Insomnia subjects tended to underestimate their TST and overestimate SOL and WASO while HS subjects overestimated their TST but had close approximation of their SOL and WASO (Table 4).

\subsection{ACT and SDs}

As shown in Table 4, all ACT and home SD parameters except TIB differentiated the insomnia and HS groups. All measures of sleep initiation and fragmentation were worse in the insomnia groups compared with controls. The PTSD group had significantly higher ACT-WASO as compared with the PTSD-mTBI group (62 (IQR 13) vs. 51(IQR 23) min, $p=0.03$, Conover-Inman's test). Insomnia and HS groups remained in bed for a similar duration but the insomnia groups slept significantly less than HSs. Although the PTSD-mTBI group had longer median TST and TIB as compared to the PTSD group, this did not reach statistical significance. There were no other significant differences in ACT measures of sleep continuity (SE, NA $\geqslant 5 \mathrm{~min}$, or fragmentation index) or in any home SD variables between the insomnia groups.

Differences were also noted in the habitual sleep times between the groups. Insomnia groups went to bed and woke up 1-2 h later than the HS group. Insomnia subjects often extended their typical weekday sleep duration on weekends by more than $2 \mathrm{~h}$ suggestive of sleep deprivation or worse insomnia during the weekdays. Daytime napping was relatively common with $33 \%$ of the PTSD and $67 \%$ PTSD-mTBI groups reporting napping at least twice weekly in SDs. Naps ranged in duration from $20 \mathrm{~min}$ to $4 \mathrm{~h}$ but most naps (56\%) lasted $1 \mathrm{~h}$ or more (Table 5 ).

\section{Discussion}

\subsection{Insomnia characterisation and clinical correlates}

Insomnia is characterised by complaints of difficulty falling or staying asleep or of early morning awakenings on most nights of the week, producing troublesome daytime symptoms. Current hypothesised pathophysiology includes the hyperarousal model where physiological and/or cognitive arousal interfere with normal sleep functioning. In addition, insomnia is thought to be perpetuated by maladaptive sleep-related behaviours which interfere with the underlying homoeostatic drive to sleep and the circadian timing of sleep. Chronic insomnia with short sleep duration is associated with important consequences including increased health-care use, workplace absenteeism, risk of hypertension, diabetes, and allcause mortality $[9-11,41]$. Identifying and treating insomnia in young veterans is paramount to prevent its harmful consequences. To this end, the objective of this study was to provide a comprehensive description of chronic insomnia and its clinical correlates in a small post-deployment clinic sample of OEF/OIF veterans with PTSD and PTSD-mTBI compared with controls. In our study, insomnia groups had greater fatigue, pain, PTSD, and depressive symptoms and overall lower HRQOL.

PTSD-mTBI subjects had similar insomnia severity and subtypes compared with PTSD subjects. Clinical variables and HRQOL were similar between the two insomnia groups with the exception of significantly greater subjective daytime sleepiness in the PTSDmTBI group. This sleepiness difference existed despite similar psychiatric co-morbidity profiles, medications and sleep durations in both insomnia groups. In addition, both groups have comparable levels of behaviourally induced sleep restriction and showed inadequate sleep hygiene. This data suggests that central causes of hypersomnia are important in this population, as has been reported in other civilian TBI populations [42-44]. Baumann et al. reported $38 \%$ of their 96 -member TBI cohort $(27 \% \mathrm{mTBI})$ had subjective post-traumatic hypersomnia 6 months after head trauma [43]. Although cerebrospinal fluid (CSF) hypocretin was found to be low acutely, most of the patients' CSF hypocretin levels returned to normal 6 months after TBI. We found a higher prevalence (60\%) of hypersomnia many years after mTBIs, making direct comparisons difficult but suggesting that hypersomnia may remain a persistent complaint. As most of our patients presented with multiple and mixed aetiology head injury, it is conceivable that damage to the hypothalamus with loss of hypocretin neurons and damage to other diffusely distributed arousal systems may have occurred as a consequence of shear stress/diffuse axonal injury sometimes seen in closed head injury [45].

\subsection{Co-morbid sleep disorders}

Despite excluding other sleep disorders (moderate-severe SDB, restless legs syndrome, and periodic limb movement disorder), comorbid sleep conditions were still prevalent in the insomnia groups. Habitual snoring associated with mild obstructive SDB events or REM sleep-related obstructive respiratory events was common (29\%) in patients with insomnia; however, we did not find differences in SDB prevalence between insomnia groups. These sleep-related respiratory events can occur in the sleep-wake transition, adding to sleep onset difficulties or precipitate middle 
Table 4

Data presented as medians (interquartile range). Post hoc significance levels are labeled as follows:

\begin{tabular}{|c|c|c|c|c|c|c|}
\hline & \multirow[t]{2}{*}{ HS (A) $(n=6)$} & \multirow[t]{2}{*}{$\operatorname{PTSD}(\mathrm{B})(n=9)$} & \multirow[t]{2}{*}{ PTSD-mTBI (C) $(n=15)$} & \multicolumn{3}{|c|}{ Cliff's delta $(\delta)^{\dagger}$} \\
\hline & & & & $\mathrm{AxB}$ & $\mathrm{AxC}$ & $\mathrm{BxC}$ \\
\hline \multicolumn{7}{|l|}{ PSG measures } \\
\hline \multicolumn{7}{|l|}{ Sleep initiation and maintenance } \\
\hline Total sleep time (min) & $386(35)^{a}$ & $318(38)$ & $309(95)$ & 0.74 & 0.1 & 0.08 \\
\hline Sleep efficiency (\%) & $96(2)^{a}$ & $85(14)$ & $90(12)$ & 0.96 & 0.47 & -0.32 \\
\hline Sleep onset latency (min) & $7(13)^{a}$ & $18(23)$ & $22(31)$ & -0.72 & -0.78 & -0.1 \\
\hline Wake after sleep onset (min) & $11(10)^{b}$ & $49(35)$ & $15(41)^{\mathrm{c}}$ & -0.85 & -0.34 & 0.46 \\
\hline No. of awakening & $6(4)^{a}$ & $17(14)$ & $13(15)$ & -0.69 & -0.68 & 0.07 \\
\hline Arousals index (arousals/h) & $1.7(0)^{\mathrm{a}}$ & $6.3(8)$ & $6.1(7)$ & -1 & -0.87 & -0.01 \\
\hline \multicolumn{7}{|l|}{ Sleep architecture } \\
\hline$\%$ of $\mathrm{N} 1$ sleep & $5(1)^{a}$ & $10(6)$ & $10(7)$ & -0.82 & -1 & -0.01 \\
\hline$\%$ of N2 sleep & $53(5)$ & $54(19)$ & $55(24)$ & -0.15 & -0.24 & -0.08 \\
\hline$\%$ of N3 sleep & $18(4)$ & $15(14)$ & $12(14)$ & 0.67 & 0.62 & 0.08 \\
\hline$\%$ of REM sleep & $21(6)$ & $19(7)$ & $18(10)$ & 0.44 & 0.56 & 0.11 \\
\hline REM latency (min) & $77(11)$ & $106(51)$ & $101(39)$ & -0.3 & -0.68 & -0.05 \\
\hline \multicolumn{7}{|l|}{ Respiratory and motor events } \\
\hline AHI (/hour) & $0.8(1)$ & $0.6(2)$ & $2.7(8)$ & 0.17 & -0.36 & -0.37 \\
\hline PLMSI (/hour) & $1.3(9)$ & $0(1)$ & $2.6(6)$ & 0.37 & -0.04 & -0.38 \\
\hline \multicolumn{7}{|l|}{ Post-PSG diary } \\
\hline Total sleep time ( $\mathrm{min}$ ) & $398(23)^{a}$ & $290(32)$ & $302(89)$ & 0.98 & 0.83 & -0.09 \\
\hline Sleep efficiency (\%) & $97(2)^{a}$ & $81(12)$ & $84(12)$ & 1 & 0.96 & -0.26 \\
\hline Sleep onset latency (min) & $8(9)^{a}$ & $33(26)$ & $30(36)$ & -1 & -0.97 & 0.05 \\
\hline Wake after sleep onset (min) & $10(9)^{\mathrm{a}}$ & $65(33)$ & $30(45)^{c}$ & -1 & -0.89 & 0.61 \\
\hline
\end{tabular}

PSG, polysomnography; AHI, apnoea-hypopnea index; PLMSI, periodic limb movements in sleep index.

a $p<0.05$ for comparison between HS and both insomnia groups;

b $p<0.05$ for comparison between HS and either PTSD or PTSD-mTBI;

c $p<0.05$ for comparisons between PTSD and PTSD-mTBI.

${ }^{\dagger}$ Effect size estimate: $\delta=0.147$ (small); $\delta=0.33$ (moderate); and $\delta=0.474$ (large).

of the night awakenings, contributing to maintenance insomnia complaints. In addition, REM and NREM sleep parasomnias were common (38\% of participants) in both insomnia groups. These may be a consequence of psychotropic medications, untreated SDB, stress, chronic sleep deprivation, or a combination of these. These findings are in accord with published increased prevalence rates of parasomnia after TBI and in psychiatric populations $[42,46]$. Nightmares, reenacting dreams concerning combat, an inherent symptom of PTSD, were significantly more common in both insomnia groups. Our results are consistent with the reported association of frequent nightmares and severe difficulties with initiating and maintaining sleep in this veteran population [47].

\section{3. $P S G$}

PSG revealed a number of differences between the insomnia groups and the HS group in sleep continuity variables including lower SE and increased SOL, awakenings and arousal index. The PTSD group also had higher WASO compared with the HS and PTSD-mTBI groups. As far as sleep architecture is concerned, both insomnia groups had increased proportional N1 sleep, a marker of greater sleep fragmentation, in accordance with previous reports in post-TBI and PTSD-associated insomniacs $[17,19]$.

\subsection{ACT/SDs}

With the exception of TIB, most sleep variables were significantly different between patients with insomnia and HS. Sleep continuity variables were worse in the PTSD group compared with PTSD-mTBI insomniacs. The PTSD-mTBI group had significantly decreased ACT-WASO as compared to the PTSD group. This pattern was also seen in the SD but did not reach statistical significance between the groups.

Our results are largely in agreement with most previous investigations employing ACT, PSG, and SDs in insomnia of differing aetiologies [18,48-50]. Although ACT WASO and TST correlated well with PSG parameters for insomniacs and HSs on one night of PSG, ACT SOL was not well correlated with PSG SOL for insomniacs. This is not surprising as PSG and ACT measure different sleep physiology constructs: the former measures EEG-defined sleep onset while the latter estimates sleep onset based on absence of movement. As compared with PSG, ACT in insomnia tends to overestimate total sleep duration and underestimate SOL, as it cannot detect motionless wakefulness [48,49].

Our ACT and home SD results corroborated many of the results of in-lab PSG. Despite some of the limitations mentioned above, we believe home ACT and SD sleep parameters are valid general estimates of the sleep of insomniacs in their home environment. A recent study in primary insomnia and normal sleepers correlating portable home PSG recordings with wrist ACT on three consecutive nights showed that estimates of WASO, TST, and SE did not differ significantly from each other across nights [50]. Medium to large correlations were found between most of the PSG and ACT sleep measures. An actigraph with a photodiode detector was useful in discovering nocturnal habits that may not have been reported otherwise (i.e., subjects sleeping with television on all night or use of portable electronic devices during awakenings). ACT may provide approximations of home sleep in insomniacs using this specific model and settings. Although SD may provide similar sleep estimations, young veterans may fail to faithfully complete SDs. Thus, ACT provides a valuable option for relatively low-cost, reliable, and objective prolonged assessment of sleep/wake patterns in the veteran's usual sleep environment.

One of the novel findings of our investigation was that significantly lower WASO was seen in the PTSD-mTBI group as compared to the PTSD group in multiple measures (PSG and home ACT). This relationship was also noted on the home SD but it did not reach statistical significance. One possible explanation for the lower PSG WASO observed in the PTSD-mTBI group is that post-traumatic hypersomnia in these subjects may partially help consolidate nighttime sleep. However, it is unclear if lower WASO in the PTSD-MTBI insomniacs is of clinical significance as both insomnia groups had similar rates of insomnia severity and subjective sleep quality. 
Table 5

Data represented as medians (interquartile range). Post hoc significance levels are labeled as follows:

\begin{tabular}{|c|c|c|c|c|c|c|}
\hline & \multirow[t]{2}{*}{ HS (A) $(n=6)$} & \multirow[t]{2}{*}{ PTSD (B) $(n=9)$} & \multirow[t]{2}{*}{ PTSD-mTBI $(C)(n=15)$} & \multicolumn{3}{|c|}{ Cliff's delta $(\delta)^{\dagger}$} \\
\hline & & & & $\mathrm{AxB}$ & $\mathrm{AxC}$ & $\mathrm{BxC}$ \\
\hline \multicolumn{7}{|l|}{ Actigraphy } \\
\hline Nights (total) & $10(1)$ & $11(5)$ & $13(3)$ & & & \\
\hline Sleep onset time & $23: 08^{b}$ & $00: 18$ & $00: 49$ & -0.63 & -0.68 & -0.16 \\
\hline Sleep offset time & $06: 27^{a}$ & 08:00 & 08:33 & -0.48 & -0.82 & -0.14 \\
\hline Time in bed (min) & $463(35)$ & $475(28)$ & $502(60)$ & -0.3 & -0.6 & -0.33 \\
\hline Total sleep time (min) & $433(37)^{a}$ & $365(45)$ & $410(49)$ & 0.82 & 0.62 & -0.42 \\
\hline Sleep efficiency (\%) & $94(2)^{a}$ & $78(6)$ & $84(6)$ & 1 & 1 & -0.35 \\
\hline Sleep onset latency (min) & $9(5)^{a}$ & $36(22)$ & $33(14)$ & -1 & -0.98 & 0.11 \\
\hline WASO (min) & $20(7)^{a}$ & $62(13)$ & $51(23)^{\mathrm{c}}$ & -1 & -1 & 0.54 \\
\hline $\mathrm{NA} \geqslant 5 \min (n)$ & $1(0.4)^{a}$ & $3(0.8)$ & $3(1.4)$ & -1 & -1 & -0.06 \\
\hline Fragmentation index $(/ \mathrm{h})$ & $20(7)^{a}$ & $46(8)$ & $36(14)$ & -1 & -0.96 & 0.42 \\
\hline \multicolumn{7}{|l|}{ Sleep diary } \\
\hline Time in bed (min) & $455(38)$ & $467(71)$ & $485(84)$ & -0.26 & -0.26 & -0.06 \\
\hline Total sleep time (min) & $428(31)^{a}$ & $339(77)$ & $399(55)$ & 0.68 & 0.76 & -0.36 \\
\hline Sleep efficiency (\%) & $95(1)^{a}$ & $72(11)$ & $77(15)$ & 1 & 1 & -0.2 \\
\hline Sleep onset latency (min) & $8(4)^{a}$ & $39(20)$ & $43(21)$ & -1 & -1 & 0.07 \\
\hline WASO (min) & $18(9)^{a}$ & $81(28)$ & $53(29)$ & -1 & -1 & 0.33 \\
\hline NA $(n)$ & $1(0.3)^{a}$ & $3(1)$ & $2(3)$ & -0.78 & -0.71 & 0 \\
\hline Sleep quality & $4(0.2)^{a}$ & $2(0.9)$ & $3(0.5)$ & 1 & 1 & -0.12 \\
\hline
\end{tabular}

WASO, wake after sleep onset; NA, nocturnal awakenings.

a $p<0.05$ for comparison between HS and both insomnia groups;

b $p<0.05$ for comparison between HS and PTSD-mTBI;

${ }^{c} p<0.05$ for comparison between PTSD and PTSD-mTBI

${ }^{\dagger}$ Effect size estimate: $\delta=0.147$ (small); $\delta=0.33$ (moderate); and $\delta=0.474$ (large)

All of the significant differences between the groups were accompanied by large effect sizes. Value distributions between groups were disparate and differences large enough to be detected despite small sample sizes.

\subsection{Insomnia aetiology}

Insomnia aetiology in this veteran population is complex and there are multiple interacting aetiologies for the sleep difficulties veterans experience upon returning to civilian life. War-specific demands increase veteran's risk for insomnia including crowded sleeping conditions, loud noise exposure, and the emergence of poor sleep habits imposed by irregular sleep-wake schedules [51]. Soldiers often sleep when they can, engaging in daytime napping, with these habits entrenched during deployments. For example, most of our insomniacs reported shift work history during military service. In a cross-sectional study of 156 deployed Air Force personnel, more than $40 \%$ of them fulfilled chronic insomnia criteria using SDs and three-quarters reported worsened sleep quality during deployment [51]. In a recent large longitudinal cohort study in this population, deployed, or post-deployed personnel reported shorter adjusted sleep duration and higher odds of sleeping difficulties compared with those who had not been deployed [13]. Those reporting mental health symptoms and combat exposure had higher odds of sleeping difficulties suggesting that these variables mediate sleeping problems during and after deployment. Furthermore, disrupted sleep following recent trauma exposure predicts the development of PTSD [22]. Upon returning home, psychosocial stressors can result in emotional arousals, tension, and rumination, adding further to sleeping difficulties.

Inadequate sleep hygiene was common in both insomnia groups, evidenced by daytime napping, excessive caffeine intake, and oversleeping on weekends. Surfing the Internet and nocturnal exercise were reported as strategies of 'exhausting' oneself to sleep. Recent studies in active military personnel have noted similar activities incongruent with sleep onset during deployment [51]. These may be reinforced after military separation by difficulties in finding schooling or employment with steady schedules. One recent multifaceted treatment trial combining cognitive behavioural therapy and image rehearsal therapy targeting sleep hygiene irregularities and co-morbid nightmares has been shown to be successful in the OEF/OIF veterans with PTSD [52].

Mood, anxiety, and substance abuse disorders may be consequences of chronic insomnia as well as potential causes of insomnia in OEF/OIF veterans [4,47]. A chart review of outpatient OEF/ OIF veterans describing the relationship between the polytrauma clinical triad (pain, PTSD, and TBI) and insomnia found that PTSD and pain, but not mTBI, were significantly associated with insomnia [4]. However, in contrast to our study, this series did note that insomnia was more severe in patients with PTSD and co-morbid mTBI. Similarly, in people who have a history of mTBI, the presence of PTSD has been shown to increase the risk for persistent postconcussive symptoms including insomnia [53]. Although subjects in whom pain was a significant contributor to insomnia were excluded, it is likely that insomnia was partially mediated by PTSD and depression symptoms.

\subsection{Study limitations}

Our study has several methodological limitations, the most important of which is using the participant's account to diagnose mTBI years after the incidents. In most cases, military medical records were not available to corroborate the history. Self-report may have resulted in misdiagnosis of $\mathrm{mTBI}$, especially in veterans with potential secondary gain. Second, in our laboratory PSG, we awakened subjects earlier than their usual home waking times, which likely affected their TST and sleep architecture. Third, participants were allowed to continue using psychotropic/hypnotic medications which affect sleep parameters; thus, some differences (or lack thereof) between the groups may have been mediated by medications. Withdrawal of these medications may have unmasked other differences between the groups as psychotropic medications (antidepressants and benzodiazepines) have varied effects on sleep architecture [54]. Hypnotic medications may have also influenced participant ability to subjectively predict sleeping abnormalities, affected overscoring of ACT TST and parasomnia prevalence [49]. However, we chose to allow medication use due to its clinical relevance and potential recruitment difficulties. Most 
insomnia subjects reported improved sleep quality during the inlab PSG, which may have also dampened existing differences. Time duration between deployment and sleep assessment makes identifying the insomnia aetiology difficult. We were unable to recruit veterans with insomnia and $\mathrm{mTBI}$ alone without any psychiatric symptoms; therefore, we could not determine the independent impact of TBI on sleep complaints. This is partially related to the fact that the Miami VA Medical Center is a third-tier trauma centre and subjects are not evaluated until about 1 year after military separation. Therefore, most patients who suffered mTBIs have recuperated by the time they enter the VA system [5]. Patients who suffered mTBI and also have psychiatric symptoms may be most likely to seek medical treatment. All participants were male so that our findings may not extend to female veterans, who often report worse insomnia symptoms [13]. Our study does not allow us to determine what percentage of insomnia is related to PTSD, depression, mTBI, or inadequate sleep hygiene established during deployment. Finally, due to limited statistical power, we cannot exclude modest differences between the groups.

Our study suggests that insomnia in OEF/OIF veterans with PTSD or PTSD-mTBI share some clinical, PSG, and ACT characteristics with those of primary insomnia. The significant differences between our insomnia groups included greater daytime sleepiness and lower PSG and ACT WASO detected in the PTSD-mTBI group. Veterans with insomnia may have a number of other sleep disorders and sleep hygiene issues, which merit multifaceted assessment and treatment. The American Academy of Sleep Medicine (AASM) does not recommend PSG as part of the routine evaluation of chronic insomnia patients but does endorse it in the setting of suspected sleep-related breathing disorder, sleep-related movement disorder, or sleep-related violent behaviours [55]. Given that these co-morbid disorders may require independent treatment, PSG should be considered in veterans with insomnia associated with PTSD and/or mTBI. ACT may be a valuable complementary tool in evaluating the sleep of young military veterans and assessing their response to pharmacologic or behavioural treatment. Our characterisation may help further define clinical approaches for insomnia treatment; however, larger studies with subjects free of psychotropic medications are needed to establish if other clinical differences may exist in military veterans with insomnia.

\section{Conflict of interest}

The ICMJE Uniform Disclosure Form for Potential Conflict of interest associated with this article can be viewed by clicking on the following link: doi:10.1016/j.sleep.2011.06.004.

The authors have no conflicts of interests regarding the project described herein.

\section{Acknowledgements}

This material is based upon work performed at the Miami VA Health Care System. The views expressed in this article are those of the authors and do not necessarily reflect the position or policy of the Department of Veterans Affairs or the United States Government.

\section{References}

[1] Hoge CW, McGurk D, Thomas JL, Cox AL, Engel CC, Castro CA. Mild traumatic brain injury in U.S. soldiers returning from Iraq. $\mathrm{N}$ Engl $\mathrm{J}$ Med 2008;358(5):453-63.

[2] McLay RN, Klam WP, Volkert SL. Insomnia is the most commonly reported symptom and predicts other symptoms of post-traumatic stress disorder in
U.S. service members returning from military deployments. Mil Med 2010;175(10):759-62.

[3] Lew HL, Poole JH, Vanderploeg RD, et al. Program development and defining characteristics of returning military in a VA Polytrauma Network Site. J Rehabil Res Dev 2007;44(7):1027-34.

[4] Lew HL, Pogoda TK, Hsu PT, et al. Impact of the "polytrauma clinical triad" on sleep disturbance in a department of veterans affairs outpatient rehabilitation setting. Am J Phys Med Rehabil 2010;89(6):437-45.

[5] Lundin A, de Boussard C, Edman G, Borg J. Symptoms and disability until 3 months after mild TBI. Brain Inj 2006;20(8):799-806.

[6] Germain A, Buysse DJ, Nofzinger E. Sleep-specific mechanisms underlying posttraumatic stress disorder: integrative review and neurobiological hypotheses. Sleep Med Rev 2008;12(3):185-95.

[7] Zeitzer JM, Friedman L, O'Hara R. Insomnia in the context of traumatic brain injury. J Rehabil Res Dev 2009;46(6):827-36.

[8] Leger D, Scheuermaier K, Philip P, Paillard M, Guilleminault C. SF-36: evaluation of quality of life in severe and mild insomniacs compared with good sleepers. Psychosom Med 2001;63(1):49-55.

[9] Vgontzas AN, Liao D, Bixler EO, Chrousos GP, Vela-Bueno A. Insomnia with objective short sleep duration is associated with a high risk for hypertension. Sleep 2009;32(4):491-7.

[10] Vgontzas AN, Liao D, Pejovic S, Calhoun S, Karataraki M, Bixler EO. Insomnia with objective short sleep duration is associated with type 2 diabetes: a population-based study. Diab Care 2009;32(11):1980-5.

[11] Vgontzas AN, Liao D, Pejovic S, et al. Insomnia with short sleep duration and mortality: the Penn State cohort. Sleep 2010;33(9):1159-64.

[12] Lew HL, Vanderploeg RD, Moore DF, et al. Overlap of mild TBI and mental health conditions in returning OIF/OEF service members and veterans. J Rehabil Res Dev 2008;45(3):11-6.

[13] Seelig AD, Jacobson IG, Smith B, et al. Sleep patterns before, during, and after deployment to Iraq and Afghanistan. Sleep 2010;33(12):1615-22.

[14] Dikmen S, McLean A, Temkin N. Neuropsychological and psychosocial consequences of minor head injury. J Neurol Neurosurg Psychiatry 1986;49(11):1227-32.

[15] Ouellet MC, Beaulieu-Bonneau S, Morin CM. Insomnia in patients with traumatic brain injury: frequency, characteristics, and risk factors. J Head Trauma Rehabil 2006;21(3):199-212.

[16] Schreiber S, Barkai G, Gur-Hartman T, et al. Long-lasting sleep patterns of adult patients with minor traumatic brain injury (mTBI) and non-mTBI subjects. Sleep Med 2008;9(5):481-7.

[17] Ouellet MC, Morin CM. Subjective and objective measures of insomnia in the context of traumatic brain injury: a preliminary study. Sleep Med 2006;7(6):486-97.

[18] Kaufman Y, Tzischinsky O, Epstein R, Etzioni A, Lavie P, Pillar G. Long-term sleep disturbances in adolescents after minor head injury. Pediatr Neurol 2001;24(2):129-34.

[19] Kobayashi I, Boarts JM, Delahanty DL. Polysomnographically measured sleep abnormalities in PTSD: a meta-analytic review. Psychophysiology 2007;44(4):660-9.

[20] Mellman TA, Nolan B, Hebding J, Kulick-Bell R, Dominguez R. A polysomnographic comparison of veterans with combat-related PTSD, depressed men, and non-ill controls. Sleep 1997;20(1):46-51.

[21] Ross RJ, Ball WA, Dinges DF, et al. Motor dysfunction during sleep in posttraumatic stress disorder. Sleep 1994;17(8):723-32.

[22] Mellman TA, Bustamante V, Fins AI, Pigeon WR, Nolan B. REM sleep and the early development of posttraumatic stress disorder. Am J Psychiatry 2002;159(10):1696-701.

[23] Breslau N, Roth T, Burduvali E, Kapke A, Schultz L, Roehrs T. Sleep in lifetime posttraumatic stress disorder: a community-based polysomnographic study. Arch Gen Psychiatry 2004;61(5):508-16.

[24] Mellman TA, Kumar A, Kulick-Bell R, Kumar M, Nolan B. Nocturnal/daytime urine noradrenergic measures and sleep in combat-related PTSD. Biol Psychiatry 1995;38(3):174-9.

[25] Krakow B, Haynes PL, Warner TD, et al. Nightmares, insomnia, and sleepdisordered breathing in fire evacuees seeking treatment for posttraumatic sleep disturbance. J Trauma Stress 2004;17(3):257-68.

[26] Westermeyer J, Sutherland RJ, Freerks M, et al. Reliability of sleep log data versus actigraphy in veterans with sleep disturbance and PTSD. J Anxiety Disord 2007;21(7):966-75.

[27] Wohlgemuth WK, Edinger JD, Fins AI, Sullivan Jr RJ. How many nights are enough? The short-term stability of sleep parameters in elderly insomniacs and normal sleepers. Psychophysiology 1999;36(2):233-44.

[28] American Academy of Sleep Medicine. The international classification of sleep disorders: diagnostic and coding manual. 2nd ed. Westchester, IL: American Academy of Sleep Medicine; 2005.

[29] Weathers FW, Keane TM. PCL-M for DSM-IV. Boston: National Center for PTSDBehavioral Science Division; 1991.

[30] Bastien $\mathrm{CH}$, Vallieres A, Morin CM. Validation of the insomnia severity index as an outcome measure for insomnia research. Sleep Med 2001;2(4):297-307.

[31] Johns MW. Reliability and factor analysis of the Epworth sleepiness scale. Sleep 1992;15(4):376-81.

[32] Kaynak H, Altintas A, Kaynak D, et al. Fatigue and sleep disturbance in multiple sclerosis. Eur J Neurol 2006;13(12):1333-9.

[33] Storch EA, Roberti JW, Roth DA. Factor structure, concurrent validity, and internal consistency of the Beck depression inventory-second edition in a sample of college students. Depress Anxiety 2004;19(3):187-9. 
[34] McHorney CA, Ware Jr JE, Raczek AE. The MOS 36-Item Short-Form Health Survey (SF-36): II. Psychometric and clinical tests of validity in measuring physical and mental health constructs. Med Care 1993;31(3):247-63.

[35] Katz DA, McHorney CA. The relationship between insomnia and health-related quality of life in patients with chronic illness. J Fam Pract 2002:51(3):229-35.

[36] Kelly JP, Rosenberg JH. Diagnosis and management of concussion in sports. Neurology 1997;48(3):575-80.

[37] Iber C, American Academy of Sleep Medicine. The AASM Manual for the Scoring of Sleep and Associated Events: Rules, Terminology and Technical Specifications. 1st ed. Westchester, IL: American Academy of Sleep Medicine; 2007.

[38] Romano J, Corragio J, Skowronek J. Appropriate statistics for ordinal level data: should we really be using $t$-test and Cohen's d for evaluating group differences on the NSSE and other surveys? In: Annual Meeting of the Florida Association of Institutional Research. Cocoa Beach, FL; 2006 p. 1-33.

[39] Meissel K. A practical guide to using Cliff's delta as a measure of effect size where parametric equivalents are inappropriate. In: ACSPRI Social Science Methodology Conference 2010. Sydney, Australia; 2010.

[40] Ledesma RD, Macbeth G, Kohan NC. Computing effect effect size measures with VISta-The Visual Statistics System. Tutorials Quant Meth Psych 2009;5(1):25-34.

[41] Thase ME. Correlates and consequences of chronic insomnia. Gen Hosp Psychiatry 2005;27(2):100-12.

[42] Verma A, Anand V, Verma NP. Sleep disorders in chronic traumatic brain injury. J Clin Sleep Med 2007;3(4):357-62.

[43] Baumann CR, Werth E, Stocker R, Ludwig S, Bassetti CL. Sleep-wake disturbances 6 months after traumatic brain injury: a prospective study. Brain 2007;130(Pt 7):1873-83.

44] Castriotta RJ, Wilde MC, Lai JM, Atanasov S, Masel BE, Kuna ST. Prevalence and consequences of sleep disorders in traumatic brain injury. J Clin Sleep Med 2007;3(4):349-56.
[45] Baumann CR, Bassetti CL, Valko PO, et al. Loss of hypocretin (orexin) neurons with traumatic brain injury. Ann Neurol 2009;66(4):555-9.

[46] Lam SP, Fong SY, Ho CK, Yu MW, Wing YK. Parasomnia among psychiatric outpatients: a clinical, epidemiologic, cross-sectional study. J Clin Psychiatry 2008;69(9):1374-82.

[47] Gellis LA, Gehrman PR, Mavandadi S, Oslin DW. Predictors of sleep disturbances in Operation Iraqi Freedom/Operation Enduring Freedom veterans reporting a trauma. Mil Med 2010;175(8):567-73.

[48] Vallieres A, Morin CM. Actigraphy in the assessment of insomnia. Sleep 2003;26(7):902-6

[49] Lichstein KL, Stone KC, Donaldson J, et al. Actigraphy validation with insomnia. Sleep 2006;29(2):232-9.

[50] Sanchez-Ortuno MM, Edinger JD, Means MK, Almirall D. Home is where sleep is: an ecological approach to test the validity of actigraphy for the assessment of insomnia. J Clin Sleep Med 2010;6(1):21-9.

[51] Peterson AL, Goodie JL, Satterfield WA, Brim WL. Sleep disturbance during military deployment. Mil Med 2008;173(3):230-5.

[52] Ulmer CS, Edinger JD, Calhoun PS. A multi-component cognitive-behavioral intervention for sleep disturbance in veterans with PTSD: A Pilot Study. J Clin Sleep Med 2011;7(1):57-68.

[53] Bryant RA, Harvey AG. Postconcussive symptoms and posttraumatic stress disorder after mild traumatic brain injury. J Nerv Ment Dis 1999;187(5):302-5.

[54] Wilson S, Argyropoulos S. Antidepressants and sleep: a qualitative review of the literature. Drugs 2005;65(7):927-47.

[55] Littner M, Kushida CA, Anderson WM, et al. Practice parameters for the role of actigraphy in the study of sleep and circadian rhythms: an update for 2002. Sleep 2003;26(3):337-41. 